\title{
Electromagnetic Interference on Artificial Heart Pacemaker
}

\author{
S. Sahrani, N. Semangin, S. Suhaili, D. A. Awg Mat, M. S. Osman, M. Sawawi \\ Faculty of Engineering, Universiti Malaysia Sarawak, 94300 Kota Samarahan, Sarawak. \\ sshafrida@feng.unimas.my
}

\begin{abstract}
Electromagnetic interference (EMI) due to Radio Frequency (RF) is known to cause interference in electronics devices. Interference may cause devices such as pacemaker to malfunctions with potentially adverse consequences. Exposure of electromagnetic interference comes from digital cellular telephones that are commonly used nowadays. This paper describes the preliminary case study on the effect of the EMI interference with a pacemaker. Temperatures were calculated at various frequencies to investigate the effect. Higher temperatures may cause discomfort to the patient.
\end{abstract}

Keywords: Electromagnetic Interference (EMI), Radio Frequency (RF), pacemaker

\section{Introduction}

Recent developments in electronics industry are major contributions to the widespread use of microwave and radio frequency (RF) devices including telecommunication, radio, radar and biomedical applications. The study of electromagnetic radiation (EMR) and human health has led to major changes in beliefs regarding the risk that may involve in modification.

Digital cellular phones have become part of our everyday life. The convenience of being able to communicate with anyone from anywhere at anytime has become part of our modern lifestyle. Most cellular phones have either a small antenna attached or integrated into internal parts. The antenna from these phones produced greater radio frequency (RF) exposure than other types of RF systems through transmit and receive data [1].

Human exposure to RF fields in excess of the limit may lead adverse health effect. The exposure to the radiation can cause heating of tissues that leads to an increase in body temperature. Although body has its own effective ways of regulating body temperature, nevertheless, if the radiation exposures are too high (over than $2 \mathrm{~Gy}$ ), the body may no longer be able to cope.

As a consequence, the induced exposure and rising thermal can cause EMI to the susceptible implanted medical devices i.e artificial heart pacemaker. The interference may cause pacemaker malfunctions and affects irritation, discomfort and an illness to patient's health. It may cause irritation and discomfort to the users.

\section{Pacemaker}

Pacemaker is a small, battery-operated electronic biomedical device that capable to normalize the human heartbeats when its natural regulating mechanisms break down [2]. It provides electric impulses that mimic the natural human heartbeats. The device is inserted under the skin to help the heart to contract and to pump blood throughout the body at an appropriate rate.

Pacemaker normally implanted in human chest cavity where its' electrode has direct contact with the heart. The electronic circuitry in pacemaker will determine a pacing pulse through a sensing device. The sensor will turns the signal off when the heartbeat is above a certain level, and turns the signal back on when the heartbeat is under par.

\subsection{Pacemaker Generator}

The pulse generator case of the pacemaker is made of titanium. It has high modulus of elasticity, high resistance to corrosion and also high durability. It also contains one to three insulated thin wires that travel through a vein in the chest to the heart.

A pacemaker generator consists of battery, circuitry and connector block. Battery supplies the electrical energy for pacing or sending of tiny electrical pulses in pacemakers. The small and lithium sealed battery typically can last from 4 to 8 years [3], depending on the active passive usage. The entire pacemaker requires complete change if the battery is low.

The electronic circuitry of pacemakers transforms the energy from the battery into tiny electrical pulses. It controls timing of the electrical pulses and amount of energy delivers to the heart. Connector block, a transparent plastic located on top of the pacemaker's metal container to connect the pacing lead with the pacemaker.

\subsection{Pacing Lead}

Pacing lead is a flexible insulated wire that connected to a pacemaker. The lead is capable to hold 